Revue de droit comparé du travail et de la sécurité sociale

3 | 2018

La participation des travailleurs dans la grande entreprise privée et publique

\title{
Le système de participation des travailleurs dans l'entreprise privée en France
}

\section{Gilles Auzero}

\section{(2) OpenEdition}

\section{Journals}

Édition électronique

URL : https://journals.openedition.org/rdctss/1916

DOI : $10.4000 /$ rdctss. 1916

ISSN : 2262-9815

Éditeur

Centre de droit comparé du travail et de la sécurité sociale

Édition imprimée

Date de publication : 1 novembre 2018

Pagination : 118-125

ISSN : 2117-4350

Référence électronique

Gilles Auzero, «Le système de participation des travailleurs dans l'entreprise privée en France », Revue de droit comparé du travail et de la sécurité sociale [En ligne], 3 | 2018, mis en ligne le 01 novembre 2021, consulté le 12 novembre 2021. URL : http://journals.openedition.org/rdctss/1916 ; DOI : https:// doi.org/10.4000/rdctss.1916

\section{(c) (i) $\ominus$}

Revue de droit comparé du travail et de la sécurité sociale est mise à disposition selon les termes de la Licence Creative Commons Attribution - Pas d'Utilisation Commerciale - Pas de Modification 4.0 International. 


\title{
LE SYSTÈME DE PARTICIPATION DES TRAVAILLEURS DANS L'ENTREPRISE PRIVÉE EN FRANCE
}

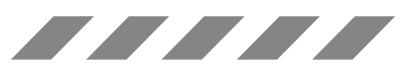

\begin{abstract}
If we confine ourselves to a formal approach, it would appear that French law has established a number of mechanisms to organise the participation of employees in the management of private companies, or, more accurately, in the legal entities that form their legal structure. While dividing this participation into two aspects, namely co-management and co-determination, the law, which governs these matters through both the Labour Code and the Commercial Code, is also meant to be coercive. Nevertheless, the fact remains that the legislator's first steps along this path have been distinctly timid. The mechanisms in question in fact include many inadequacies that mean that employee participation in the management of private companies has to be considered, if not as an edifice still to be constructed, then at least as a work in progress.
\end{abstract}

KEYWORDS : Employee Participation, Legal Entity, Company, Co-management, co-determination, Labour Law, Corporate Law.

\section{RÉSUMÉ}

A s'en tenir à une approche formelle, il apparaît que le Droit français institue un certain nombre de dispositifs organisant la participation des salariés à la gestion de l'entreprise privée ou, plus exactement, de la personne morale qui, juridiquement, la structure. Déclinant cette participation sous ses deux versants que sont la cogestion et la codécision, les textes de loi, qui relèvent tout à la fois du Code du travail et du Code de commerce, se veulent qui plus est contraignants. II n'en demeure pas moins que le législateur n'a emprunté cette voie que de manière bien timide. Les dispositifs en cause souffrent, en effet, de nombreuses insuffisances qui conduisent à considérer que la participation des salariés à la gestion de l'entreprise privée reste encore largement, sinon à construire, du moins à parfaire.

MOTS CLÉS : Participation des salariés, personne morale, société, cogestion, codécision, droit du travail, droit des sociétés. 


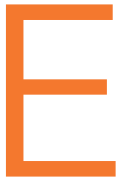

n France, le terme de " participation » est diversement utilisé. II revêt un sens étroit quand est en cause la participation des salariés aux résultats dans l'entreprise. Dans cette hypothèse, on a coutume de parler de participation financière. II a un sens plus large qui, dans notre hiérarchie des normes, est consacré au plus haut niveau, celui des principes constitutionnels. Ainsi que le précise l'alinéa 8 du Préambule de la Constitution du 27 octobre 1946, dont on sait qu'il fait partie intégrante de notre bloc de constitutionnalité, "Tout travailleur participe, par l'intermédiaire de ses délégués, à la détermination collective des conditions de travail ainsi qu'à la gestion des entreprises ».

Ce texte fondamental englobe tout à la fois le droit des salariés à la négociation collective et la participation à la gestion, c'est-à-dire aux décisions. Sans doute est-il fait référence, dans les deux cas, à la participation des travailleurs. Pour autant, seule la participation à la gestion sera retenue par la suite car elle seule implique une association des salariés à la prise de décision. Tel n'est pas l'objet de la négociation collective et de ses fruits, les conventions et accords collectifs de travail. Ainsi que l'énonce l'article L. 2221-1 du Code du travail, ces normes ont pour objet de fixer l'ensemble des conditions d'emploi, de formation professionnelle et de travail des salariés, ainsi que leurs garanties sociales. Partant, la négociation collective ne se saisit pas, à proprement parler, des décisions susceptibles d'être prises par l'employeur. Elle ne matérialise pas un partage du pouvoir ; elle accompagne le pouvoir.

A s'en tenir donc à la participation à la gestion, celle-ci peut recouvrir deux dispositifs distincts. Le premier, que l'on qualifiera de cogestion, recouvre les règles assurant une présence des représentants des salariés dans les organes sociaux de la société employeur. Le second renvoie à la codécision, dont il résulte que certaines décisions ne peuvent être prises sans le consentement des représentants du personnel, ces derniers se voyant alors conférer un droit de veto.

Jusqu'à une date relativement récente, le Droit français n'a ménagé qu'une place très résiduelle à la participation à la gestion ainsi entendue. S'agissant tout d'abord de la présence de représentants des salariés dans les organes sociaux de la société employeur, elle était abandonnée à la libre volonté des associés¹. En effet, ainsi que le précise, en

1 On doit cependant préciser que dans les sociétés cotées en Bourse, lorsque les salariés détiennent au moins $3 \%$ du capital social de la société, un ou plusieurs administrateurs sont élus par l'assemblée générale parmi les salariés actionnaires ou, le cas échéant, parmi les salariés membres du conseil de surveillance d'un fonds commun de placement d'entreprise détenant des actions de la société (C. com., art. L. 225-33). Pour être salariés ces administrateurs représentent moins ces derniers qu'une catégorie particulière d'actionnaires. Pour cette raison essentielle le dispositif ne sera pas envisagé ici. 
substance, les articles L. 225-27 et L. 225-79 du Code du commerce, « il peut être stipulé dans les statuts » que le conseil d'administration ou le conseil de surveillance comprend des administrateurs ou membres élus par le personnel. Le dispositif est ainsi triplement limité. Outre, comme il a été dit, que sa mise en œuvre exige une stipulation statutaire, il suppose que la société employeur ait adopté une forme sociale impliquant l'existence d'un conseil d'administration ou d'un conseil de surveillance. Enfin, et en tout état de cause, les représentants des salariés, dont il faut tout de même relever, qu'ils disposent d'une voix délibérative, restent toujours en minorité, par l'application même de la loi.

Quant à la codécision, elle était et, nous le verrons, reste quasiment inexistante. II n'est en effet guère besoin de rappeler que le comité d'entreprise n'est, à de très rares exceptions près, doté que d'attributions consultatives. En d'autres termes, dès lors que l'employeur a pris soin de solliciter son avis, il reste maître de la décision. La prochaine disparition du comité d'entreprise, dont les attributions seront exercées par un comité social et économique, regroupant par ailleurs les délégués du personnel et le comité d'hygiène, de sécurité et des conditions de travail ne change pas fondamentalement la donne. Mais, nous y reviendrons, la réforme semble vouloir ménager une certaine place à la codécision sans véritablement l'imposer.

Il apparaît ainsi que certains changements sont à l'œuvre dans le système juridique français. Ils ont d'abord concerné, en 2013, la cogestion. Ayant enfin pris conscience que la liberté avait ici ses limites, les dispositions précitées du Code de commerce ayant été très peu mises en œuvre, le législateur a fait le choix de la contrainte, imposant la présence de représentants des salariés dans les organes sociaux des sociétés employeurs. Mieux vaut dire de certaines sociétés car, nous y reviendrons, la réforme est d'une portée très limitée. Mais le saut qualitatif n'en reste pas moins réel. Cela mérite d'être d'autant plus souligné que dans une importante décision en date du 26 juillet 2013, curieusement restée relativement inaperçue chez les juristes de Droit du travail, le Conseil constitutionnel a pu affirmer, sans guère d'explication, que « le huitième alinéa du Préambule de 1946 n'impose pas la présence de représentants des salariés au sein des organes de direction de l'entreprise $»^{2}$.

On notera qu'un approfondissement du dispositif était attendu des ordonnances réformant le Code du travail. La loi d'habilitation autorisait en effet le Gouvernement à prendre des mesures « améliorant les conditions de représentation et de participation des salariés dans les organes d'administration et de surveillance des sociétés dont l'effectif dépasse certains seuils ». Sauf à avoir mal lu les ordonnances, cette habilitation n'a pas été suivie d'effets, sans que l'on puisse expliquer pourquoi.

Il n'en demeure pas moins que le droit positif assure d'ores et déjà une certaine participation des salariés à la gestion, aux moyens de dispositifs que nous présenterons dans un premier temps (I), avant d'en souligner, dans un second temps, les insuffisances (II).

2 Cons. const., 26 juill. 2013, n² 2013-333 QPC, Dr. sociétés, nov. 2013, comm. 179, obs. D. GalloisCochet. Une autre position, plus exigeante, aurait pu être retenue.

3 Art. $2,4^{\circ}$ de la loi d'habilitation. 


\section{I - LES DISPOSITIFS EXISTANTS}

Laissant de côté les dispositifs purement facultatifs, qui n'ont trouvé que de trop rares expressions concrètes, nous nous concentrerons sur les dispositifs obligatoires tendant à assurer une participation des salariés à la gestion. En reprenant la distinction établie précédemment, nous envisagerons successivement la codécision (A) et la cogestion (B).

\section{A - LA CODÉCISION}

Dans la mesure où il est, en fait, exclu de conférer un pouvoir de codécision à l'ensemble des salariés d'une entreprise, celui-ci ne peut qu'être attribué à une institution représentative du personnel élue par eux. Le moins que l'on puisse dire est que le législateur français n'a, jusqu'à ce jour, guère exploré cette voie, préférant multiplier à l'envi les hypothèses dans lesquelles, avant de prendre une décision, l'employeur doit seulement recueillir l'avis du comité d'entreprise.

On trouve néanmoins trace, dans le Code du travail, de très rares cas dans lesquels le comité d'entreprise se voit doter d'un véritable de droit de veto. II en va ainsi en matière $\mathrm{d}^{\prime}$ horaires individualisés ${ }^{4}$, de la nomination du médecin du travail ${ }^{5}$ ou encore de la proposition de postes de reclassement interne à des salariés menacés d'un licenciement pour motif économique ${ }^{6}$. Chacun appréciera la part du pouvoir de décision de l'employeur qui se trouve ainsi soumise à l'aval du comité d'entreprise.

Une évolution pourrait survenir par l'effet d'une des ordonnances de réforme du Code du travail. L'ordonnance relative à la nouvelle organisation du dialogue social et économique dans l'entreprise et favorisant l'exercice et la valorisation des responsabilités syndicales emporte au premier chef une fusion des institutions représentatives du personnel élues dans l'entreprise. A brève échéance le comité social et économique se substituera aux délégués du personnel, au comité d'entreprise et au comité d'hygiène de sécurité et des conditions de travail.

Toutefois, un accord collectif d'entreprise ou de branche peut muer ce comité social et économique en conseil d'entreprise qui, en sus des prérogatives conférées par la loi au premier, est compétent pour, sauf exceptions légales, négocier et conclure des conventions et accords collectifs de travail. Or, en application du futur article L. 2320-2 du Code du travail, l'accord collectif instituant le conseil d'entreprise doit fixer « la liste des thèmes tels que l'égalité professionnelle, soumis à l'avis conforme du conseil d'entreprise ». C'est donc bien de codécision dont il est ici question et, bien que le texte ne soit pas parfaitement clair, il est fait obligation d'ouvrir certains «thèmes » à celle-ci. Car ce n'est pas une obligation de négocier qui est en cause ici, mais bien une obligation de conclure. On relèvera d'ailleurs que le texte précité fait de la formation un «thème obligatoire ". En d'autres termes, si conseil d'entreprise il y a, la formation devra nécessairement être soumise à l'accord de celui-ci.

4 C. trav., art. L. 3121-48, qui évoque « l'avis conforme du comité d'entreprise ».

5 C. trav., art. R. 4623-5 qui évoque «l'accord du comité d'entreprise ».

6 C. trav., art. R. 1233-45-1, qui évoque « l'avis favorable du comité d'entreprise ». 
Il apparaît au total que le développement de la codécision dans notre droit positif est abandonné à la volonté des partenaires sociaux, c'est-à-dire à un accord de l'employeur et des syndicats de salariés. Il est loin d'être certain que le premier accepte ainsi librement de se déposséder d'une partie de son pouvoir de décision. Gageons dès lors qu'en fait de conseil d'entreprise, c'est le comité social et économique qui dominera et, avec lui, la concertation plutôt que la codécision.

\section{B - LA COGESTION}

On doit à la loi relative à la sécurisation de l'emploi du 14 juin 2013 d'avoir imposé la présence de représentants des salariés dotés d'une voix délibérative au sein des conseils d'administration et de surveillance. Si la contrainte est réelle, elle doit néanmoins être relativisée, compte tenu d'abord du champ d'application de l'obligation en cause.

En effet, l'élection de représentants des salariés au sein du conseil d'administration ou du conseil de surveillance n'est imposée que dans les sociétés qui emploient, à la clôture de deux exercices consécutifs, au moins mille salariés permanents dans la société et ses filiales, dont le siège social est fixé sur le territoire français, ou au moins cinq mille salariés permanents dans la société et ses filiales, dont le siège social est fixé sur le territoire français et à l'étranger ${ }^{7}$. Eu égard à leur importance, les seuils d'effectif limitent, par hypothèse, le champ d'application du dispositif. Encore faut-il souligner qu'ils ont été abaissés par la loi Rebsamen du 17 août 2015 puisqu'ils étaient antérieurement et respectivement de 5000 et 10000 salariés! On remarquera toutefois que, nonobstant la formule légale regrettable, ce n'est pas tant l'effectif d'une société qui est prise en compte que celui du groupe auquel elle appartient. Pour le dire autrement, une société anonyme employant 60 salariés sera soumise à l'obligation légale dès lors qu'elle est la filiale d'un groupe qui emploie plus de 1000 salariés en France.

Alors même que cette condition serait remplie, la mise en œuvre du dispositif légal apparaît à géométrie variable. En effet, ainsi que le précise le Code de commerce, une société n'est pas soumise à l'obligation de faire siéger des représentants des salariés dans ses organes sociaux dès lors qu'elle est la filiale, directe ou indirecte, d'une société ellemême soumise à cette obligation ${ }^{8}$. Ce que l'on peut traduire de la manière suivante : si dans un groupe, la société mère assure la présence de représentants des salariés au sein de son conseil d'administration ou de son conseil de surveillance, l'ensemble de ses filiales échappe à cette même obligation...

La règle est, si l'on peut dire, inversée lorsque la société mère est une société holding qui emploie moins de cinquante salariés ${ }^{9}$. Dans ce cas, elle peut ne pas mettre en œuvre l'obligation légale si elle détient une ou plusieurs filiales remplissant les conditions et appliquant cette même obligation.

Notons enfin que lorsqu'une société est tenue de faire siéger des représentants des salariés dans ses organes sociaux, ces derniers, pour disposer d'une voix délibérative, seront en minorité. Il résulte en effet de la loi que le nombre d'administrateurs ou de

7 C. com., art. L. 225-27-1 et L. 225-79-2.

8 C. com., art. L. 225-27-1 et L. 225-79-2.

9 Ou plus précisément, pour reprendre les termes de la loi, « une société dont l'activité principale est d'acquérir et de gérer des filiales et des participations". 
membres du conseil de surveillance représentant les salariés est au moins égal à deux dans les sociétés dont le nombre d'administrateurs ou de membres plus « classiques » est supérieur à douze et au moins un s'il est égal ou inférieur à douze.

En définitive, il apparaît clairement que la présence de représentants des salariés dans les organes sociaux des entreprises privées doit être relativisée. Il faut à nouveau regretter que le Gouvernement n'ait pas remis l'ouvrage sur le métier lors de l'écriture des ordonnances de réforme du Code du travail.

Il est en revanche un dispositif obligatoire d'application plus générale. De longue date, le Code du travail permet au comité d'entreprise de déléguer deux de ses membres pour participer à toutes les séances du conseil d'administration ou du conseil de surveillance. La délégation peut être portée à quatre membres ou, au contraire, être réduit à un membre, notamment si l'organe social compte au moins un représentant des salariés en vertu du dispositif précédemment étudié ${ }^{10}$. Cette limitation peut être discutée, d'une part, parce que les représentants du comité d'entreprise n'ont qu'une voix consultative, ce qui n'est donc pas de nature à rompre les équilibres au sein de l'organe de direction et, d'autre part, parce qu'il n'y a pas d'identité entre des représentants élus par les salariés et des représentants désignés par une institution représentative du personnel.

\section{II - UN DISPOSITIF INSUFFISANT}

Les développements qui précèdent ont d'ores et déjà laissé entrevoir un certain nombre d'insuffisances du dispositif français de participation des salariés à la gestion de l'entreprise à laquelle ils appartiennent. Mais il est d'autres insuffisances, sans doute plus profondes et insidieuses, qui touchent à l'insuffisante prise en compte de la forme sociale adoptée par l'employeur, d'une part (A), et à celle du fonctionnement sociétaire d'autre part (B).

\section{A - L'INSUFFISANTE PRISE EN COMPTE DE LA FORME SOCIALE}

Lorsque j'ai présenté les dispositifs de cogestion obligatoire, il a beaucoup été question de la participation des salariés ou des représentants du comité d'entreprise aux conseils d'administration et de surveillance. Or, parmi les différentes formes sociales offertes par le droit français pour juridiquement structurer une entreprise, seules les sociétés anonymes et les sociétés en commandite par actions sont obligatoirement dotées de tels organes sociaux. Si les secondes sont, historiquement, en nombre extrêmement réduit, les premières voient leur nombre constamment diminuer depuis l'avènement de la société par actions simplifiées (SAS). Les chiffres officiels en témoignent. L'INSEE révèle ainsi qu'en 2016, 40 \% des sociétés créées étaient des SARL et $56 \%$ des SAS. Les $4 \%$ restant concernent toutes les autres sociétés, qu'il s'agisse des SA, des SCA, des SCS, des sociétés civiles, etc. ${ }^{11}$ !

La SARL, pas plus que la SAS, ne sont tenues de mettre en place un conseil d'administration ou un conseil de surveillance. Sans doute peuvent-elles le faire volontairement, par une stipulation de leurs statuts. Mais, même dans ce cas, il s'avère qu'elles ne seront pas soumises aux dispositifs de cogestion obligatoire. Plus précisément, il résulte de la loi elle-même que ces sociétés ne sont soumises à l'obligation de faire siéger

10 C. trav., art. L. 2323-65.

11 Insee Première, $n^{\circ}$ 1631, janv. 2017. 
des représentants élus par les salariés dans les organes sociaux, quand bien même elle comporterait un conseil d'administration ou un conseil de surveillance ${ }^{12}$.

Il faut donc se rendre à l'évidence et constater que ces sociétés et spécialement la SAS, qui connaît un net succès en pratique et peut juridiquement organiser des entreprises d'une taille conséquente, reste en marge du dispositif obligatoire de participation. En d'autres termes, il suffit pour éviter les rigueurs de la loi à cet égard d'opter pour cette forme sociale... Heureusement, les sociétés cotées en Bourse ne peuvent, dans notre système juridique, qu'adopter la forme d'une société anonyme.

Pour en revenir à la SAS, il était à dire vrai difficile de la soumettre au dispositif de participation des salariés institués par le Code de commerce. En effet, celui-ci ne peut se concevoir qu'en présence d'organes sociaux collégiaux au sein desquels les représentants des salariés vont siéger. Or, en vertu de la loi, doit seul être obligatoirement mis en place dans ces sociétés un président. Mais, à tout le moins, aurait-il été envisageable que le Code de commerce étende le dispositif en cause aux SAS qui, volontairement, se dotent d'un organe collégial.

Il est à remarquer que, s'agissant de la participation de représentants du comité d'entreprise aux conseils d'administration ou de surveillance des sociétés, l'article L. 232366 du Code du travail dispose que « dans les sociétés par actions simplifiées, les statuts précisent l'organe social auprès duquel les délégués du comité d'entreprise exercent les droits définis par la présente sous-section ». Dès lors que la société ne comporte qu'un président, la rédaction de la clause statutaire sera aisée : ce sera auprès de lui que les représentants du comité exerceront leurs prérogatives. Mais, leur exercice ne s'en trouvera pas facilité. Ce n'est pas la même chose de participer à un organe collégial et de se retrouver dans une sorte de tête-à-tête avec une seule et même personne. Mais c'est déjà là aborder les insuffisances de la loi au regard du fonctionnement sociétaire.

\section{B - L'INSUFFISANTE PRISE EN COMPTE DU FONCTIONNEMENT SOCIÉTAIRE}

Imposer une participation à la gestion, c'est, à l'évidence, imposer un partage du pouvoir. Partant, cela implique nécessairement de s'interroger sur le détenteur de ce pouvoir. De prime abord, il est tentant de retenir qu'il s'agit des organes sociaux, c'est-àdire le conseil d'administration et le conseil de surveillance. Mais c'est oublier que, dans une société, le pouvoir est d'abord détenu par les associés, dont les dirigeants ne sont que les mandataires. Cela est exacerbé par l'extraordinaire financiarisation de l'économie, qui contribue à donner de larges prérogatives aux actionnaires; que la théorie de la corporate governance a d'ailleurs contribué à renforcer.

Bien qu'il existe un certain nombre de modalités, plus ou moins insidieuses, qui permettent à un actionnaire, singulièrement lorsqu'il est majoritaire, de mettre en œuvre son pouvoir sur une société, celui-ci est d'abord, et au moins formellement, exercé au sein des assemblées générales. Or, le Droit n'organise pas l'accès des salariés aux assemblées générales, du moins lorsqu'ils ne sont par ailleurs actionnaires.

II faut cependant faire mention de l'article L. 2323-67 qui, dans un troisième alinéa, dispose que « deux membres du comité d'entreprise, désignés par le comité (...) peuvent

12 L'article L. 227-1 du Code de commerce exclut purement et simplement l'application du dispositif en cause aux SAS. 


\section{PARTICIPATION DANS L'ENTREPRISE PRIVÉE EN FrANCE}

assister aux assemblées générales. Ils sont entendus, à leur demande, lors de toutes les délibérations requérant l'unanimité des associés ». Cette disposition, qui assure la présence de représentants des salariés au sein des assemblées générales de manière très indirecte, apparaît nettement insuffisante. Tout d'abord, si les délégués du comité peuvent assister à toutes les assemblées générales, qu'elles soient ordinaires ou extraordinaires, ils n'ont droit à la parole que de façon extrêmement ponctuelle. Le droit de s'exprimer ne leur est en effet conféré que pour les délibérations soumises à l'unanimité. Or, sans entrer dans le détail de la question, on soulignera simplement que la loi n'exige cette unanimité que dans des hypothèses marginales, par exemple le changement de nationalité ou lorsque la délibération a pour objet d'accroître les obligations des associés.

Ensuite, et il faut y revenir, il n'existe pas nécessairement d'assemblées générales des associés au sein des SAS. Sans doute, les statuts peuvent-ils en prévoir, mais cela n'est guère le cas en pratique.

Pour en revenir au comité d'entreprise, l'article L. 2323-67 lui octroie d'autres prérogatives, qui ne sont pas inintéressantes. En effet, ce texte prévoit que, « dans les sociétés, le comité d'entreprise peut demander en justice la désignation d'un mandataire chargé de convoquer l'assemblée générale des actionnaires en cas d'urgence. Il peut également requérir l'inscription de projets de résolutions à l'ordre du jour des assemblées ». Le dispositif est tout à fait intéressant en ce qu'il oblige les actionnaires à se saisir de questions à l'initiative du comité d'entreprise. Mais encore faut-il que ces questions relèvent des prérogatives de l'assemblée générale... En outre, en serait-il ainsi que cela n'autorise pas les représentants du comité à s'exprimer devant les actionnaires.

Au final, il ressort de tout cela que le législateur français a su organiser quelques dispositifs de nature à assurer un véritable partage du pouvoir, en permettant aux salariés ou à leurs représentants de quitter la sphère de l'entreprise pour pénétrer celle de la personne morale qui l'organise juridiquement et qui est titulaire des pouvoirs qui la prennent pour objet. Mais ce même législateur est intervenu a minima. Partant, il serait sans doute souhaitable qu'il remette l'ouvrage sur le métier afin d'accroître les droits des salariés au titre de leur participation à la gestion de l'entreprise. Mais, de mon point de vue, une telle réforme ne peut avoir pour objet le seul Droit du travail. C'est une vision globale qu'il convient d'adopter en réformant celui-ci, mais aussi le Droit des sociétés. Or, et cela relève de l'évidence, il y a là un important facteur de blocage.

\section{GILLES AUZERO}

Professeur à I'Université de Bordeaux, membre du Comptrasec, UMR 5114 CNRS.

Thèmes de recherche : Droit du travail, Relations collectives de travail, Rapports Droit du travail et Droit des sociétés.

\section{Publications :}

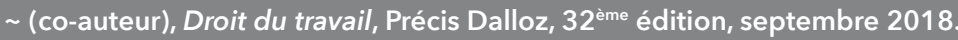

$\sim$ "Les transformations du comité social et économique », JCP S, 2018, 1227.

$\sim$ " Accords collectifs et entreprises à structures complexes », Droit ouvrier, 2017, p. 365.

"Le pouvoir de l'employeur dans les groupes de sociétés : un pouvoir sous influence " : Droit des sociétés, 2017, p. 19. 